\title{
On the nature of the $30-\mu \mathrm{m}$ feature in carbon-rich Planetary Nebulae
}

\section{F. Kemper}

Academia Sinica, Institute of Astronomy \& Astrophysics, Taiwan

E-mail: ciska@asiaa.sinica.edu.tw

\section{Otsuka}

Academia Sinica, Institute of Astronomy \& Astrophysics, Taiwan

\section{J. Cami}

University of Western Ontario, Canada

SETI Institute, USA

\section{E. Peeters}

University of Western Ontario, Canada

SETI Institute, USA

\section{J. Bernard-Salas}

Open University, $U K$

We report on the analysis of the $30-\mu \mathrm{m}$ feature seen in the spectra of ten $C_{60}$-containing carbonrich Galactic planetary nebulae, and establish that the feature and the continuum emission could be due to the same carrier.

The Life Cycle of Dust in the Universe: Observations, Theory, and Laboratory Experiments 18-22 November 2013

Taipei, Taiwan

\footnotetext{
* Speaker.
} 
We have searched the Spitzer Space Telescope archive for Galactic Planetary Nebulae observed with the Infrared Spectrograph (IRS), showing the characteristic resonances due to $\mathrm{C}_{60}$ at 17.4 and $18.9 \mu \mathrm{m}$ [1]. We identified 11 such sources, including five that were previously known to contain fullerenes. The spectra all show a prominent $30-\mu \mathrm{m}$ feature, commonly attributed to MgS [2]. However, doubt was cast over this identification by Zhang et al. [3], using energetic and abundance arguments.

We have explored the possibility that the $30-\mu \mathrm{m}$ feature is an intrinsic resonance to the carrier of the cold dust continuum, by constructing an astronomical carbon-dust opacity table (Fig. 1), and fitting it to ten of the sources in our sample for which sufficient spectral data are available. We are able to achieve a good fit in all ten cases (Fig. 2), implying that the carrier of the dust continuum and the $30-\mu \mathrm{m}$ feature can be one and the same material. Indeed, the graphite opacities presented by Draine \& Lee [4] show a strong resonance around $40 \mu \mathrm{m}$, which may shift to shorter wavelengths when the conductivity of the graphite changes [5].

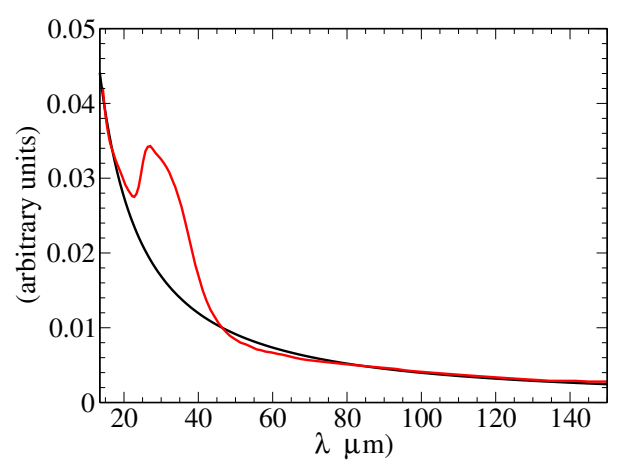

Figure 1: The derived opacity table for the carrier of the $30 \mu \mathrm{m}$ feature and the dust continuum (red), compared to a power-law opacity $\propto \lambda^{-1.2}$ (black). Figure taken from [1].

These results recently appeared in press, and the reader is referred to Section 4.8 in the work by Otsuka et al. [1], where a detailed description of the analysis is given.

\section{References}

[1] M. Otsuka, F. Kemper, J. Cami, E. Peeters, \& J. Bernard-Salas, Physical properties of fullerene-containing Galactic planetary nebulae, MNRAS 437, 2577-2593 (2014), [1310 . 7711 ]

[2] S. Hony, L. B. F. M. Waters, \& A. G. G. M. Tielens, The carrier of the "30" $\mu$ m emission feature in evolved stars. A simple model using magnesium sulfide, A\&A 390, 533-553 (2002), [astro-ph/0204330]

[3] K. Zhang, B. W. Jiang, \& A. Li, On Magnesium Sulfide as the Carrier of the $30 \mu \mathrm{m}$ Emission Feature in Evolved Stars, ApJ 702, 680-685 (2009), [0 907 . 1037]

[4] B. T. Draine, \& H. M. Lee, Optical properties of interstellar graphite and silicate grains, ApJ 285 , 89-108 (1984)

[5] B. Jiang, A. Li, J. Liu, J. Gao, \& A. Mishra, Unidentified species in envelopes around evolved stars, in Diffuse Interstellar Bands, eds. J. Cami \& N. Cox, IAU Symposium 297 (2013) , [1307. 4014 ] 


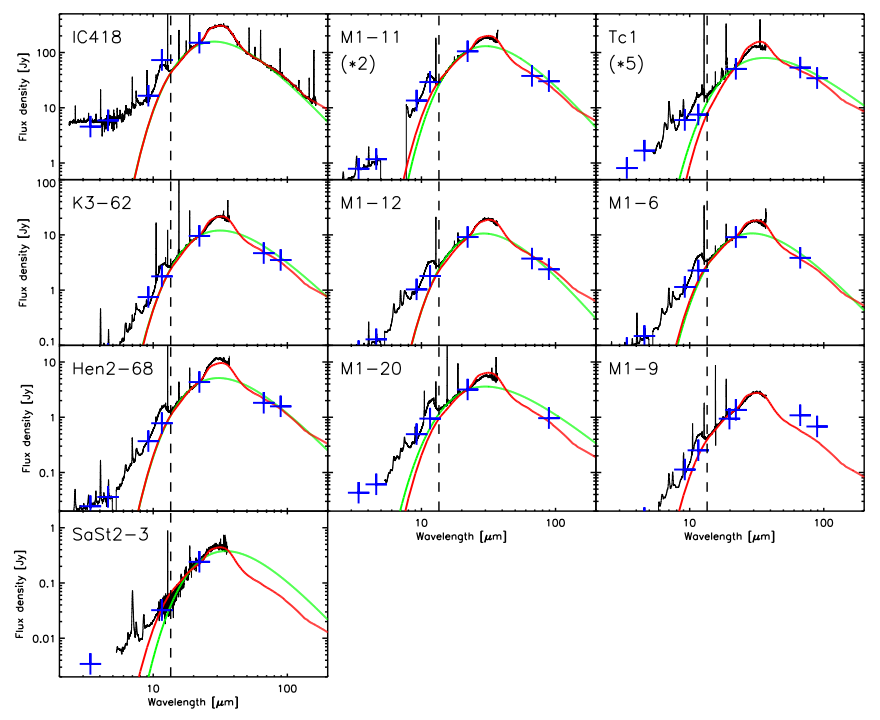

Figure 2: The best fit results for each of the objects to the continuum emission beyond $13.5 \mu \mathrm{m}$, and the $30 \mu \mathrm{m}$ feature. The spectra are shown in black, and the photometry points with blue crosses. The red line shows the best fit result, which was fit to the data to the right of the dashed line. The best fit modified blackbody, e.g. a single temperature blackbody multiplied by a $\lambda^{-p}$ emissivity law, to the same data is shown for comparison in each of the panels with a green line. For clarity, the spectra of M1-11 and Tc 1 are shifted by the indicated factor. Figure taken from [1]. 